\title{
Rare Case of Bilateral Coexistence of Caroticoclinoid Foramen, Inter- Clinoid Bar and Posterior Clinoid Foramen in a Dry Human Skull
}

\author{
Syed Rehan Hafiz Daimi ${ }^{1}$, Radi Ali M. Alsaffar ${ }^{2}$
}

${ }^{1}$ Department of Anatomy, College of Medicine, Imam Abdulrahman Bin Faisal University, Kingdom of Saudi Arabia. ${ }^{2}$ Department of Anatomy, College of Medicine, Imam Abdulrahman Bin Faisal University, Kingdom of Saudi Arabia.

\section{INTRODUCTION}

The superior surface of the body of the sphenoid is centrally occupied by sella turcica. The sella turcica has median depression called as hypophysial fossa; its anterior border is formed by tuburculum sella and posterior by dorsum sellae. It is surrounded by two anterior and posterior clinoid processes at its corners. Some parts of the sphenoid bone are connected by the ligaments that occasionally ossify. ${ }^{1}$ These ligaments have important relations with the surround vascular and neuronal structures. Hence such altered anatomy leads to difficulties or serious complications during surgical approach of this region in various conditions like aneurysm of internal carotid artery (ICA), caroticoclinoid fistula, pituitary adenoma and tuberculum sellae meningiomas etc. Knowledge of such variations would help radiologists and neurosurgeons in accurate diagnosis and treatment of the patients. Caroticoclinoid foramen (CCF) is an inconstant or abnormal anatomical feature formed due to ossification of a ligament between anterior and middle clinoid processes. It was first described by Henle (1855) as quoted by Mallik S and Sawant VG. ${ }^{2}$ It was postulated that although ossification of the ligaments is normally age dependent physiological condition but CCF is an exception as its existence is seen even in the fetal and infant skull because of developmental anomaly. ${ }^{3}$ The development of the sphenoid bone is complex has 18 or 19 ossification centers which may lead to early ossification of carotico-clinoid ligament. ${ }^{4}$

CCF allows the passage of clinoid segment of the internal carotid artery. ${ }^{5}$ It may lead to compression, tightening or stretching and cause changes in the artery which reflects in compression of the cavernous sinus ${ }^{6}$. CCF may also cause endocrinal problems affecting hypothalamus or hypophysis cerebri and neurological problems by affecting oculomotor nerve, optic nerve or pituitary gland, which manifest in different ways as headache, behavioural changes, hormonal disturbances, visual problems, epileptic seizers, craniofacial disorders and tooth anomalies. ${ }^{7}$ Interclinoid osseous bar (ICB) results from ossification of the interclinoid ligament present between anterior and posterior clinoid processes. ${ }^{8}$ The osseous bridges represent a developmental anomaly of the embryonic chondrocranium. ${ }^{4}$ The ICB can be partial or complete; unilateral or bilateral. ${ }^{2}$ ICB may influence structures like trochlear nerve, oculomotor nerve, pituitary gland or remains as a normal variant. ICB is demonstrated radiologically in idiots, criminals, epileptics and cases of mental disorders. ${ }^{2,8}$

Posterior clinoid foramen (PCF) is very rare and scarcely mentioned in the literature. Jolly Agarwal and Virendra Kumar recorded it in one specimen unilaterally (2.5\%). PCF, when present exists on the sphenoid bone posterior to CCF. It is created between middle and posterior clinoid processes when the interclinoid bar is complete (i.e. between anterior and posterior clinoid processes). ${ }^{9}$
Corresponding Author: Syed Rehan Hafiz Daimi, Department of Anatomy, College of Medicine, Imam Abdulrahman Bin Faisal University, Dammam, Kingdom of Saudi Arabia. E-mail: srdaimi@iau.edu.sa

DOI: $10.14260 /$ jemds/2020/13

Financial or Other Competing Interests: None.

How to Cite This Article:

Daimi SRH, Alsaffar RAM. Rare case of bilateral coexistence of caroticoclinoid foramen, inter-clinoid bar and posterior clinoid foramen in a dry human skull. J. Evolution Med. Dent. Sci. 2020;9(01):60-62, DOI: $10.14260 /$ jemds/2020/13

Submission 03-11-2019,

Peer Review 19-12-2019,

Acceptance 27-12-2019,

Published 06-01-2020. 
CCF is an underestimated structure, which has significant neuronal and vascular relations which make it both clinically and surgically important. ${ }^{4}$ Although the occurrence of CCF and ICB is not rare, the modern and conventional anatomy textbooks does not mention about such variations. However the only source of information can be accessed through the published articles. The aim of present study is to document the morphology and morphometry of CCF, PCF and ICB and to consider the possible effect on surrounding neurovascular structures.

\section{PRESENTATION OF CASE}

During routine osteology teaching class in the Department of Anatomy, a dry human skull with triple osseous variations in the sphenoid bone was detected. The approximate estimated age and sex of the skull is not known. There were no morphological and pathological changes in the surrounding bone. The following osseous variations were noted in the skull-

1. Bilateral complete CCF was detected in the sphenoid bone. The shape of CCF was circular with a diameter of $5 \mathrm{~mm}$ on both sides. (Figure 1)

2. The interclinoid bar (ICB) was noted bilaterally between anterior and posterior clinoid processes of the sphenoid bone. The width of ICB measured was $6 \mathrm{~mm}$ at the beginning and $6 \mathrm{~mm}$ in the middle and $7 \mathrm{~mm}$ at the end. Its complete length from anterior to posterior clinoid processes was $14.7 \mathrm{~mm}$ on right side and $14.3 \mathrm{~mm}$ on left side.

3. A foramen was observed posterior to $\mathrm{CCF}$, it was named as "posterior clinoid foreman" (PCF). PCF was located bilaterally between middle and posterior clinoid processes. It was oval shaped, and the diameter recorded was $4 \mathrm{~mm}$ transversely and $3 \mathrm{~mm}$ vertically on both sides. (Figure 2)

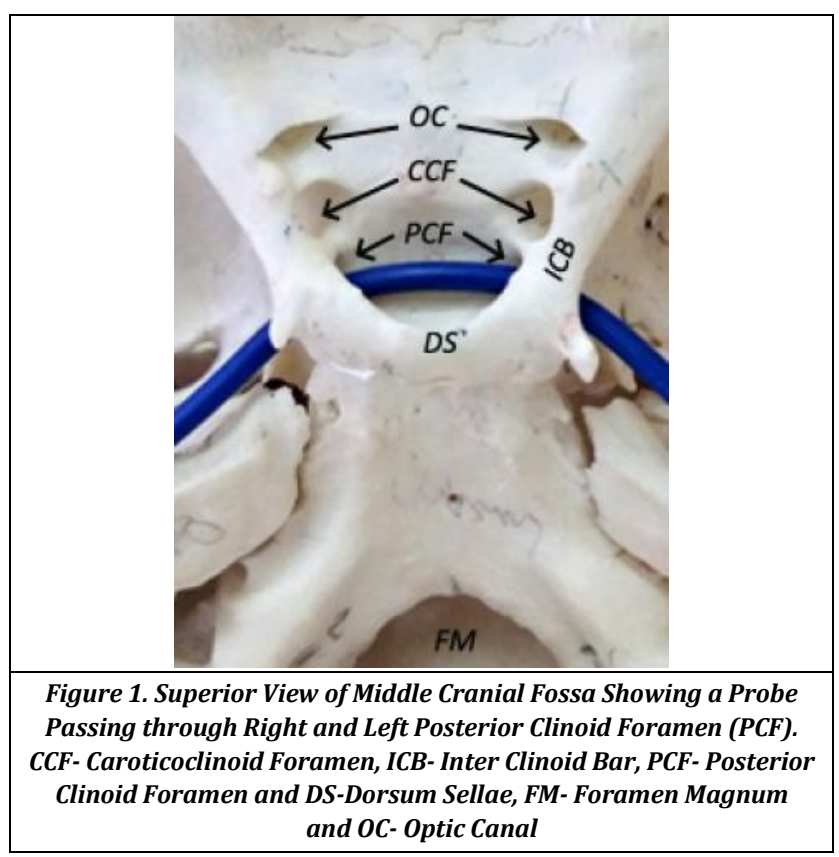

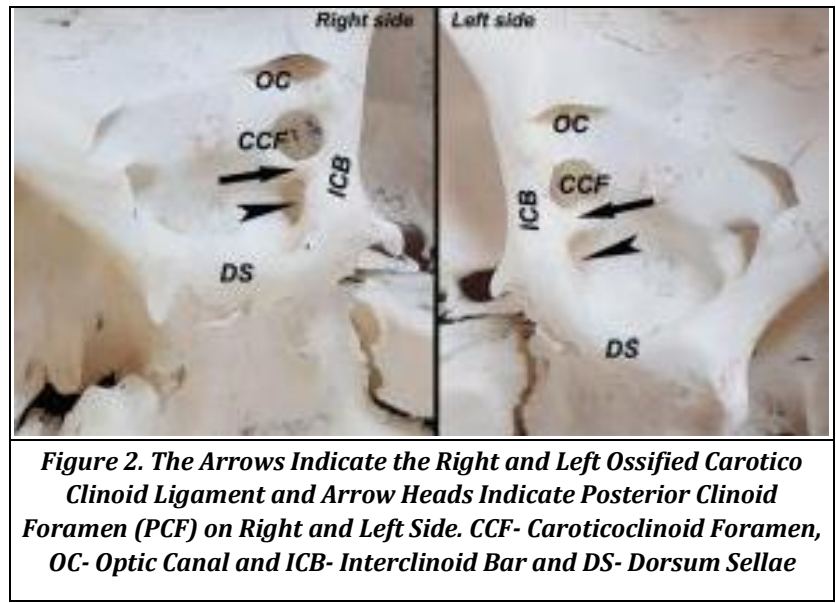

\section{DISCUSSION}

Ossification of the ligaments is age related physiological process. Ossification of caroticoclinoid ligament and interior clinoid ligament are not rare and reported frequently in the literature. However, the triple combination of the osseous variations including CCF, ICB and PCF described in this case, to the best of our knowledge has never been reported. Various studies has been conducted on dry skull as well as on fresh autopsy specimen and found that, the incidence of CCF and ICB varies in different populations worldwide. ${ }^{2} \mathrm{~A}$ racial variation has also been reported regarding CCF, which shows highest incidence in Turkish population (35-67\%) followed by Japanese (9.9\%) and Brazilians (2.5\%). ${ }^{6}$ In addition it was found more frequent on the right side $(9.2 \%)$ than on the left $(5.5 \%)^{10}$. Moreover regarding the sexual variations, reports were contradictory. Lee et al. documented it more common in males, while Freire et al. found its frequency higher in the females. ${ }^{10,5}$

Keyers has classified the CCF into three types; complete, contact and incomplete. whereas the Rani et al. has classified it into 4 types: Type 1 - Bridge present between anterior and middle clinoid process; Type 2 - Bridge between the anterior, middle and posterior clinoid process; Type 3 - Bridge between anterior and posterior clinoid process and Type 4 - Bridge between the middle and posterior clinoid process. ${ }^{11,12}$ As per Keyers classification the CCF foramen in present case is of complete type as bony bridge was found between anterior and middle clinoid processes and according to Rani et al. classification it fall in type $-2^{\text {nd }}$ category.

Ozdomus et al. revealed the average diameter of the CCF on the right side was $5.14 \mathrm{~mm}$ whereas on left side it was 5.25 $\mathrm{mm}$. Erturk et al. has documented transverse diameter of the foramen as $5.32 \mathrm{~mm} .4,8$ In the present case the diameter recorded was $5 \mathrm{~mm}$ on both sides, in accordance with the observation made by above authors. It was reported by Ozdogmus et al. that when the caliber of the ICA is larger than diameter of CCF, there is a high possibility of headache due to atrial compression. ${ }^{4}$ The incidence of interclinoid osseous bridge by various authors as quoted by Erturk et al are; $8.68 \%$ by Keyers, $2.22 \%$ by Azeredo et al. $4 \%$ by Inoue et al., $2 \%$ by Cireli et al., $4.54 \%$ by Deda et al., and $1.01 \%$ by Gurun et al. ${ }^{8}$ Malik and Sawant reported the width of anterior, middle and 
posterior part of the ICB as $6.6 \mathrm{~mm}, 2.36 \mathrm{~mm}$ and $4.1 \mathrm{~mm}$ respectively. ${ }^{2}$ In the present case it was about $6 \mathrm{~mm}$ at the beginning and $6 \mathrm{~mm}$ in the middle and $7 \mathrm{~mm}$ at the end. The length of ICB revealed by Agarwal and kumar was $11.90 \mathrm{~mm}$ and in the present case it was $14.7 \mathrm{~mm}$ on right side and 14.3 mm on left side. ${ }^{9}$

The posterior clinoid foramen (PCF) which was located on posterior aspect of CCF i.e. between middle and posterior clinoid processes was present bilaterally and its shape was oval on both the sides in present study. Its diameter was $4 \mathrm{~mm}$ transversally and $3 \mathrm{~mm}$ vertically. The diameter of PCF was smaller as compare to CCF. Agarwal and kumar documented unilateral presence of $\mathrm{PCF}$ in $2.5 \%$, they recorded its transverse diameter as $3.05 \mathrm{~mm} .{ }^{9}$ It would be difficult to comment about what structure would passes through PCF as it was observed in the dry skull. According to its location it may be concluded that either posterior intercavernous sinus or a branch of basilar venous plexus would transmit through it. It is just an assumption which needs to be confirmed by detail cadaveric studies or radiological investigations in the living subjects.

The existence of CCF can produce compression, stretching or tightening of internal carotid artery which gives rise to headache and other neuronal symptoms due to insufficient blood supply to the brain. ${ }^{13,4}$ During management of proximal ICA aneurysms, anterior clinoid process is removed to expose structures of the cavernous sinus. The presence of CCF and ICB may increase the risk of rupture or tear of ICA, leading to fatal cerebral infarction. Preoperative recognition of the CCF by imaging studies has great clinical significance when approaching surgical lesions in the region. ${ }^{8} \mathrm{CCF}$, ICB and PCF have a great clinical significance and knowledge of such structures may help neurosurgeons, neurophysicians, endocrinologist, radiologist, anatomists and biological anthropologist.

\section{CONCLUSIONS}

This case presents morphology and morphometry of CCF, PCF and ICB. The PCF described in this case is rarely mentioned in the literature. As pituitary fossa is crucial from the neurosurgical aspect, any osseous variations in this region may lead to difficulties during surgical procedures. Understanding the variability of the morphological features of the sphenoid bone is integral for better surgical approach and for diagnosis and management of various related conditions in the middle cranial fossa. It would be also useful to neurophysicians, endocrinologists, radiologists, anatomists and biological anthropologists.

\section{REFERENCES}

[1] Williams PL, Warwick R, Dyson M, et al. Gray's Anatomy. 37th edn. Edinburgh: Churchill Livingstone 1989: p. 3737.

[2] Mallik S, Sawant VG. Bilateral "Carotico-clinoid foramen" with "sella Turcica Bridge"- a case report. Anat Physiol: Current Research 2015;5:S5.

[3] Kier EL. Embryology of the normal optic canal and its anomalies. An anatomic and roentgenographic study. Invest Radiol 1966;1(5):346-62.

[4] Ozdogmus O, Saka E, Tulay C, et al. The anatomy of the carotico-clinoid foramen and its relation with the internal carotid artery. Surg Radiol Anat 2003;25(3-4):241-6.

[5] Freire AR, Rossi AC, Prado FB, et al. Caroticoclinoid foramen in human skulls: incidence, morphometry and its clinical implications. Int J of Morphol 2011;29(2):427-31.

[6] Bansode SA, Devadas P, Vinila BHS. Study on the incidence of the carotico-clinoid foramen in the south Indian dry adult skulls: a cross-sectional study. International Journal of Anatomy and Research 2017;5(3.1):4051-5.

[7] Haldar A, Tirpude A, Tirpude P. Bilateral carotico-clinoid foramen in adult human skull and its surgical implication. IJAPB 2017;4(9):6-9.

[8] Erturk M, Kayalioglu G, Govsa F. Anatomy of the clinoidal region with special emphasis on the caroticoclinoid foramen and interclinoid osseous bridge in a recent Turkish population. Neurosurg Rev 2004;27(1):22-6.

[9] Agarwal J, Kumar V. Study of ossified clinoid ligaments in sphenoid bone of north Indian skulls. Journal of the Anatomical Society of India 2015;(Suppl 64):S7-S11.

[10] Lee HY, Chung IH, Choi BY, et al. Anterior clinoid process and optic strut in Koreans. Yonsei Medical Journal 1997;38(3):151-4.

[11] Keyers JEL. Observations on four thousand optic foramina in human skulls of known origin. Arch Ophthalmol 1935;13(4):538-68.

[12] Archana R, Anita R, Jyoti C, et al. Incidence of osseous interclinoid bars in Indian population. Surg Radiol Anat 2010;32(4):383-7.

[13] Purohit BJ, Singh PR. Incidence, anatomy and clinical significance of carotico-clinoid foramen and interclinoid osseous bridge in human skulls in Gujarat region. International Journal of Anatomy, Radiology and Surgery 2018;7(2):A033-A07. 